\title{
Consensus-based Distributed Optimal Energy Management with Less Communication in a Microgrid
}

DOI:

10.1109/TII.2018.2871562

\section{Document Version}

Accepted author manuscript

Link to publication record in Manchester Research Explorer

\section{Citation for published version (APA):}

Zhao, T., Li, Z., \& Ding, Z. (2018). Consensus-based Distributed Optimal Energy Management with Less Communication in a Microgrid. IEEE Transactions on Industrial Informatics, 1-1.

https://doi.org/10.1109/TII.2018.2871562

\section{Published in:}

IEEE Transactions on Industrial Informatics

\section{Citing this paper}

Please note that where the full-text provided on Manchester Research Explorer is the Author Accepted Manuscript or Proof version this may differ from the final Published version. If citing, it is advised that you check and use the publisher's definitive version.

\section{General rights}

Copyright and moral rights for the publications made accessible in the Research Explorer are retained by the authors and/or other copyright owners and it is a condition of accessing publications that users recognise and abide by the legal requirements associated with these rights.

\section{Takedown policy}

If you believe that this document breaches copyright please refer to the University of Manchester's Takedown Procedures [http://man.ac.uk/04Y6Bo] or contact uml.scholarlycommunications@manchester.ac.uk providing relevant details, so we can investigate your claim.

\section{OPEN ACCESS}




\title{
Consensus-based Distributed Optimal Energy Management with Less Communication in a Microgrid
}

\author{
Tianqiao Zhao, Zhenhong Li, Zhengtao Ding Senior Member, IEEE
}

\begin{abstract}
In this paper, to reduce required capacities for information exchanges in microgrids, a novel distributed eventbased algorithm is proposed for optimal energy management in a microgrid. Aiming at optimally scheduling the energy supplier's generation, an objective function is formulated to minimize the total cost of maintaining the supply-demand balance with considering power losses. Regarding each participant as an agent, the proposed algorithm is implemented in a distributed manner based on a multi-agent system framework. Therefore, each agent only exchanges information with its neighbours through a local network. Additionally, comparing with the periodical communication of sampled-data mechanisms, the adopted event-based scheme achieves satisfactory performance by using significantly less communication between participants. As a result, it further facilitates the development of networked microgrids. Furthermore, concerning the privacy of participants, the proposed algorithm is implemented without exposing owners' private preferences. The effectiveness of the proposed distributed algorithm is validated through several simulation studies.
\end{abstract}

Index Terms-Distributed optimization, optimal energy management, event-based mechanism, microgrids, multi-agent system

\section{INTRODUCTION}

$\mathbf{T}$ HE microgrid provides a promising solution to integrate diversely distributed generators (DGs), energy storage systems (ESSs), loads and control devices, which brings a more flexible and efficient power grid [1]. Microgrids can be operated in different modes, namely grid-connected mode and islanded mode. For a grid-connected microgrid, the balance of supply and demand can be adjusted by the main grid by absorbing power from or injecting power into the main grid [2]. In contrast, the main task of an islanded microgrid is to maintain the supply-demand balance by itself in a costeffective way. However, with high-level integration of the intermittent renewable generation and various load demands, it brings new challenges to the traditional energy management system [3].

In general, the energy management problem is formulated as an optimization problem that takes a vital part in the scheduling during the microgrid operation [4]. Typically, the energy management problem is addressed by the centralized approaches [5]-[8]. However, relying on a control centre, these

This work was supported by the Science and Technology Facilities Council under Grant ST/N006852/1

T. Zhao, Z. Li, Z. Ding are with the School of Electrical and Electronic Engineering, University of Manchester, Sackville Street Building, Manchester M13 9PL, UK (e-mails: tianqiao.zhao@manchester.ac.uk; zhenhong.li@postgrad.manchester.ac.uk; zhengtao.ding@manchester.ac.uk). centralized methods require a costly communication system to gather the system-wide information and also a powerful central controller to process a mass of received data [9], which results in an increasing susceptibility to single-point failures. Furthermore, certain sensitive information has been passed to the central state, despite the unwillingness of the participants [10].

To address such adverse effects of the centralized approaches, distributed algorithms with lower communicational and computational burdens and higher robustness are promising alternatives for the future smart grids [11]-[19]. In [13], an incremental cost consensus algorithm based multi-agent system (MAS) is proposed to solve the energy management problem. However, it relies on a leader agent to collect the current generation information so that it is not fully distributed. Similarly, the authors in [14] proposed a consensus-based algorithm to allocate active power among participants economically that deploys an external leader to process the mismatch information. In [15], a novel consensus-based algorithm is proposed to solve the economic dispatch problem in a fully distributed approach without considering the power losses. Furthermore, in [16], under the premise of maximizing the social welfare, the energy management problem is solved by a MAS-based distributed algorithm. In [17], the authors propose a continuous-time distributed algorithm for maximizing the satisfaction and welfare of plug-in electric vehicle's users without certain initialization process, which requires the sharing information about consumer's cost function. The authors in [18] introduce a distributed algorithm for economic dispatch problem in smart grids with wind power, which does not verify the algorithm under time-varying renewable generation. In addition, a distributed energy management system in [19] is introduced to energy internet by using consensus-based distributed alternating direction method of multipliers algorithm without considering the limitation of the communication network.

Regarding to address the energy management problem in a distributed manner, the consensus-based optimization algorithms utilize the communication between spatially-connected DGs and further perform the computation locally. With deploying the large-scale information technology infrastructure in the future smart grid [20], the network resources would be exhausted by the tremendous data exchange [21]. Ignoring the communication bottlenecks could lead to unreliable operations and even the failed performance of distributed approaches. Besides, agents in the future MAS would execute the algorithm 
in an embedded microprocessor that will interact with neighbours under limited computational capacities. Therefore, it is worthwhile to design a distributed algorithm with less communication requirements. From this perspective, the event-based mechanism provides a solution for a significant reduction of the information exchanges [22], [23]. Recently, the authors in [24] firstly introduce the event-triggered distributed optimization algorithm to solve the economic dispatch problem, and significantly reduce the communication burdens. However, the impacts of intermittent renewable generation have not been considered. Since the proposed algorithm also requires the initial power allocation starts from a predetermined value, the algorithm may require the re-initialization when the operation condition is changed. Furthermore, the authors in $[25]^{1}$ propose an event-triggered based energy management system for multi-energy systems to maximize their day-ahead social welfare and smooth out the real-time loads variations. However, the algorithm requires each agent to exchange two auxiliary variables between neighbours, which might result in additional communication cost.

Most of the aforementioned algorithms on energy management system require a continuous or periodical communication. Once the communication link is congested, those algorithms may fail to converge to the solution [24]. In light of energy management system over a networked environment, it is indispensable to design a distributed solution with less communication burdens. From this perspective, an event-based distributed algorithm is proposed for the optimal energy management while taking the intermittent renewable generation and load demands into consideration to maintain the supply-demand balance. Regarding each participant in the microgrid as an agent, agents in the MAS only need to exchange information through a local communication network. Thus, the proposed solution can be applied in a distributed manner to address the issues caused by centralized methods. In general, the periodic communication schemes need to take the worst situations into consideration for determining sampled periods. Therefore, those schemes may cause a waste of the communication resources. In contrast, an even-triggered mechanism is involved in developing the proposed algorithm that can further reduce communication requirements among agents dramatically. As a result, it is more compatible with future networked microgrids. Compared with [24], the event condition proposed in this paper is in a distributed manner. Besides, respecting the privacy of the individual participant, the proposed algorithm solves the problem without sharing the participant's preferences. Several simulation studies are carried out for validating the effectiveness and the scalability of the proposed algorithm.

The remainder part of the paper is organized as follows. Section II outlines the multi-agent system architecture and gives a description in the conception of the smart grid. Section III formulates the energy management in a microgrid. Section IV proposes an event-based distributed optimal solution for the energy management problem. Section V presents the simulation results and corresponding analysis. Finally, the

\footnotetext{
${ }^{1}$ appeared online after the second run of the review of this submission
}

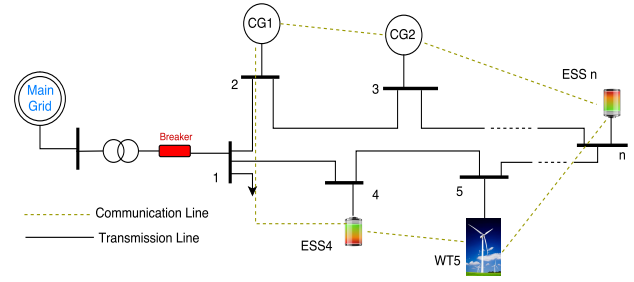

Fig. 1. The topology of a microgrid

conclusion is drawn in Section VI.

\section{Multi-Agent System in Smart Grids}

Considering a microgrid as shown in Fig. 1, a MAS framework is first introduced in the context of smart grids to take advantage of the Information and Communication Technology infrastructure in smart grids [26]. In this framework, each participant in the microgrid is regarded as an agent, which can interact with its adjacent agents to achieve certain objectives. As indicated in [27], the design of the communication network can be independent of the power network, which can be constructed according to the requirement of a practical microgrid. Hence, consider the communication network $\mathcal{G}=(\mathcal{V}, \mathcal{E})$ associated with $N$ units including both generation and demand units, where $\mathcal{V}=\left\{\nu_{1}, \ldots, \nu_{n}\right\}$ denotes the agent set and $\mathcal{E}$ is the edge set. The communication graph is undirected because of the two-way information flow of the advanced ICT infrastructure. It is assumed that there exists a path between every pair of distinct units so that the graph is connected. The adjacency matrix $\mathcal{A}=\left[a_{i j}\right] \in \mathcal{R}^{N \times N}$ of the graph $\mathcal{G}$ is defined such that $a_{i i}=0, a_{i j}=1$ if $\left(\nu_{j}, \nu_{i}\right) \in \mathcal{E}$ and $a_{i j}=0$ otherwise. The Laplacian matrix $\mathcal{L}$ associates with $\mathcal{G}$ is defined as $\mathcal{L}_{i i}=\sum_{j \neq i} a_{i j}$ and $\mathcal{L}_{i j}=-a_{i j}, i \neq j$. The degree of $i$ th agent is represented by $d_{i}=\mathcal{L}_{i i}$.

\section{Optimal Energy Management Problem}

The optimal energy management problem in this paper is first formulated as a social welfare maximization (SWM) problem that can stimulate the participants in a smart grid to be more willing to involve in the grid operation. In general, the SWM problem is defined as an optimization problem that to discover an power allocation among the participants to maximize the total welfare of the participants [28], [29],

$$
\begin{aligned}
\operatorname{Max} & \left(\sum_{(i, D) \in \mathbb{D}} W_{i, D}\left(P_{i, D}\right)+\sum_{(i, R) \in \mathbb{R}} W_{i, R}\left(P_{i, R}\right)\right. \\
& \left.+\sum_{(i, G) \in \mathbb{G}} W_{i, G}\left(P_{i, G}\right)+\sum_{(i, S) \in \mathbb{G}} W_{i, S}\left(P_{i, S}\right)\right)
\end{aligned}
$$

where $\mathbb{G}, \mathbb{S}, \mathbb{R}$ and $\mathbb{D}$ are the sets of conventional generators (CGs), energy storage systems (ESSs), renewable generators (RGs) and load demand (LDs). $W_{i, D}, W_{i, G}, W_{i, s}$ and $W_{i, R}$ are the welfare functions of load demand (LDs), conventional generators (CGs), energy storage systems (ESSs) and renewable generators (RGs), respectively. $P_{i, G}, P_{i, S}$ and $P_{i, R}$ are the active power generated from $(i, G)$ th $\mathrm{CG},(i, S)$ th ESS 
and $(i, R)$ th $\mathrm{RG}$, respectively. $P_{i, D}$ is the power consumed by $(i, D)$ th LD.

Following [30], [31], we first denote $\eta_{i, G}=\frac{\partial P_{l o s s}}{P_{i, G}}, \eta_{i, S}=$ $\frac{\partial P_{l o s s}}{P_{i, S}}, \eta_{i, R}=\frac{\partial P_{l o s s}}{P_{i, R}}, \eta_{i, D}=\frac{\partial P_{l o s s}}{P_{i, D}}$ and $\eta_{i, E V}=\frac{\partial P_{l o s s}}{P_{i, E V}}$ as the power loss efficiency of each type of participants. The different generation units are classified into two aspects, i.e., dispatchable and non-dispatchable, according to their generation types. Then welfare functions can be defined according to the conception of microgrids as follows.

\section{A. Welfare Functions of Dispatchable Units}

1) Conventional Generators: For a give price signal at time $t, \rho(t)$, the welfare for CGs can be defined as the net profit,

$$
W_{i, G}\left(P_{i, G}\right)=\rho(t)\left(1-\eta_{i, G}\right) P_{i, G}-C_{i, G}\left(P_{i, G}\right)
$$

where $\eta_{i, G}$ is the power loss efficiency of $(i, G)$ th CG defined later. $C_{i, G}\left(P_{i, G}\right)$ is the generation cost of $(i, G)$ th $\mathrm{CG}$ for providing $\left(P_{i, G}\right)$, which is written as a conventional quadratic function such as

$$
C_{i, G}\left(P_{i, G}\right)=\frac{1}{2} a_{i, G} P_{i, G}^{2}+b_{i, G} P_{i, G}+c_{i, G}
$$

where nonnegative constants $a_{i, G}, b_{i, G}$ and $c_{i, G}$ are the cost coefficients.

2) Energy Storage Systems: Similar to CGs, ESSs are regarded as dispatchable units since they are able to absorb the excessive power and compensate the insufficient power according current grid requirements. Referring to [32], it assumes that the renewable generation are insufficient to supply total demand. Therefore, the remaining demands should be supplied by the ESSs and CGs economically. From this perspective, the welfare function for each ESS is modelled as the revenue of participating in the grid regulation,

$$
W_{i, S}\left(P_{i, S}\right)=\rho(t)\left(1-\eta_{i, S}\right) P_{i, S}-C_{i, S}\left(P_{i, S}\right)
$$

where $\eta_{i, S}$ is the power loss efficiency of $(i, S)$ th ESS. $C_{i, S}\left(P_{i, S}\right):=C_{i, S}^{B 1}\left(P_{i, S}\right)+C_{i, S}^{B 2}\left(P_{i, S}\right)$ is the cost of $(i, S)$ th ESS respecting to its energy efficiency and degradation cost. Following [33], a quadratic function is introduced to indicate the cost according to charging/discharging efficiency,

$$
C_{i, S}^{B 1}\left(P_{i, S}\right)=\frac{1}{2} a_{i, S} P_{i, S}^{2}+b_{i, S} P_{i, S}
$$

where $a_{i, S}$ and $b_{i, S}$ are positive cost coefficients of $(i, S)$ th ESS.

The degradation cost of battery cells during batteries' charging/dicharging cycles plays a key factor in the optimal operation of BESSs. A linearized approximation [34] for battery degradation is adopted in this work. Taking the normalized cost of power draw from/to the battery, the battery degradation cost is given by

$$
C_{i, S}^{B 2}\left(P_{i, S}\right):=\rho_{c} \times\left|P_{i, S}\right|
$$

where $\rho_{c}(\$ / \mathrm{kW})$ is battery cost per unit power discharged/charged, and the time-slot duration is included in $\rho_{c}$.

Remark 3.1: As shown in [16], [33], similar quadratic models of BESSs have been used to indicate the the operating cost respecting to the efficiency of BESSs. Indeed, the battery cannot be charged or discharged at a same time. In this paper, following the same assumption in [16], we assume the renewable generation are insufficient to supply total demand. Therefore, the objective of this paper is to design an energy management system that schedules ESSs and CGs to support the demand in an economical approach.

\section{B. Welfare Functions of Non-dispatchable Units}

1) Renewable Generators: The forecasting generation of the RGs including WTs and PVs may not be accurate because of their the stochastic and intermittent nature so that they are considered as non-dispatchable units. Noting that the environmental policy and concerns in many countries follow the priority of using the renewable generation whenever it is available [30]. Therefore, for the optimization purpose, it is reasonable to assume that the cost of $(i, R)$ th $\mathrm{RG}$ is constant as $C_{i, R} \bar{P}_{i, R}$, since its generation cannot be adjusted during the optimization. To this end, the welfare is defined by

$$
W_{i, R}\left(P_{i, R}\right)=\rho(t)\left(1-\eta_{i, R}\right) \bar{P}_{i, R}-C_{i, R} \bar{P}_{i, R}
$$

where $\eta_{i, R}$ is the power loss efficiency of $(i, R)$ th RG, and $\bar{P}_{i, R}$ is the non-dispatchable renewable generation.

2) Constant Load Demands: The welfare for the constant LDs are modelled as the utility minus energy cost. The utility of LDs is assumed to be constant as $U_{i, D} \bar{P}_{i, D}$, since the consumed power will not be changed [29]. Therefore, the welfare of LDs is defined by

$$
W_{i, D}\left(P_{i, D}\right)=U_{i, D} \bar{P}_{i, D}-\rho(t)\left(1+\eta_{i, D}\right) \bar{P}_{i, D}
$$

where $\eta_{i, D}$ is the power loss efficiency of $(i, D)$ th LD, and $\bar{P}_{i, D}$ is the constant consumed power.

\section{Problem Formulation}

In an islanded microgrid, the mismatch between the summation of all the generated active power and the overall consumed power should be zero to maintain the system stability,

$$
\sum_{i, G \in \mathbb{G}} P_{i, G}+\sum_{i, S \in \mathbb{S}} P_{i, S}+\sum_{i, R \in \mathbb{R}} P_{i, R}=\sum_{i, D \in \mathbb{D}} P_{i, D}
$$

Next, by substituting (2) - (4) in (1), and with power balance constraint (9) and power loss efficiency, the SWM problem can be further written as

$$
\begin{array}{rr}
\operatorname{Min} & \left(\sum_{(i, G) \in \mathbb{G}} C_{i, G}\left(P_{i, G}\right)+\sum_{(i, S) \in \mathbb{S}} C_{i, S}\left(P_{i, S}\right)\right) \quad(10 \mathrm{a}) \\
\text { s.t. } & \sum_{(i, D) \in \mathbb{D}}\left(1+\eta_{i, D}\right) P_{i, D}=\sum_{(i, G) \in \mathbb{G}}\left(1-\eta_{i, G}\right) P_{i, G} \\
+\sum_{(i, S) \in \mathbb{S}}\left(1-\eta_{i, S}\right) P_{i, S}+\sum_{(i, R) \in \mathbb{R}}\left(1-\eta_{i, R}\right) P_{i, R} \\
P_{i, G}^{m} \leq P_{i, G} \leq P_{i, G}^{M} & (10 \mathrm{~b}) \\
P_{i, S}^{m} \leq P_{i, S} \leq P_{i, S}^{M} & (10 \mathrm{c})
\end{array}
$$

where (10c) - (10d) are the power limits for the generation/consumption of participants. The constant terms, i.e. the 
cost/utility of RGs/LDs, are neglected since they will not affect the optimization decision. In addition, RGs cannot be considered as dispatchable units if they are controlled in maximum peak power tracking (MPPT) mode. Therefore, only ESSs and CGs are responsible for maintaining the balance between the supply and demand.

As a result, the original problem is formulated as an optimal problem that is to schedule the usage of all the dispatchable units and minimize the generation cost while taking into consideration unpredictable LDs and RGs. For convenience of the problem formulation, we further denote $P_{i}$ as the power generation, $\eta_{i}$ as the power loss efficiency, and $P_{i}^{m}$ and $P_{i}^{M}$ are the lower and upper bound of $i$ th unit, respectively. Then (10) can be rewritten as

$$
\begin{array}{ll}
\text { Min } & \sum_{i \in N} C_{i}\left(P_{i}\right) \\
\text { s.t. } & \sum_{i \in N} \varepsilon_{i} P_{i}=P_{m} \\
& P_{i}^{m} \leq P_{i} \leq P_{i}^{M}
\end{array}
$$

where $P_{m}$ is the mismatch between the supply and demand caused by the intermittent renewable generations and varying LDs, i.e., $P_{m}=\sum\left(1+\eta_{i, D}\right) P_{i, D}-\sum\left(1-\eta_{i, R}\right) P_{i, R}$ and $\varepsilon_{i}=\left(1-\eta_{i}\right)$ of $i$ th unit.

\section{Augmented Lagrangian Function}

The objective function is firstly augmented by the KKT multiplier to find the solution set

$$
L=\sum_{i \in N} C_{i}\left(P_{i}\right)+\theta\left(\sum_{i \in N} \varepsilon_{i} P_{i}-P_{m}\right)
$$

where $\theta$ is the KKT multiplier associated with the equality constraint (11b). Here, we do not consider the KKT multiplier for the local inequality constraint since it can be restricted in the local feasible domain by applying projection methods [33].

Then the optimization problem (11) has a solution if and only if there exists a constant $\theta^{*}$ such that

$$
\begin{array}{r}
\nabla C_{i}\left(P_{i}^{*}\right)+\theta^{*} \varepsilon_{i}=0 \\
\varepsilon_{1} P_{1}^{*}+\varepsilon_{2} P_{2}^{*}+\cdots+\varepsilon_{N} P_{N}^{*}-P_{m}=0 .
\end{array}
$$

\section{E. Pricing Mechanism}

Assuming the participant in the smart grid is selfish, then the social welfare is maximized when

$$
\frac{\partial W_{i, G}\left(P_{i, G}\right)}{\partial P_{i, G}}=\frac{\partial W_{i, S}\left(P_{i, S}\right)}{\partial P_{i, S}}=0 .
$$

Adopting (2) and (4) in (14), it shows that (14) is equivalent to

$$
\frac{\partial C_{i}\left(P_{i}\right)}{\partial P_{i}}+\theta\left(1-\eta_{i}\right)=0, \quad \text { for } \quad i \in N
$$

which means $\theta$ will be interpreted as the price signal for each participant to maximize its individual welfare/benefit.

The formulated problem can be solved by a traditional centralized method, which requires all the information from participants to process the optimization algorithm. Alternatively, a proper designed distributed method is able to solve the above problem without the central communication. However, both two methods require the continuous communication, which are designed without considering the capability of the communication network. In what follows, an event-based distributed optimization algorithm is proposed to solve the above problem with taking the communication issue into account.

\section{Event-BASEd Distributed SOlution FOR THE ENERGY MANAGEMENT PROBLEM}

Since the communication infrastructure in a smart grid usually has a limited bandwidth and energy resources, agents embedded with distributed algorithms to update the optimization algorithm simultaneously, due to the communication constraints. From this perspective, in this section, an eventbased distributed algorithm will be proposed to solve the formulated optimization problem, so that the communication burden can be reduced significantly.

Before proposing our algorithm, some conventions are firstly introduced. Let $\tilde{\theta}_{j}$ be the last known information of $j$ th agent transmitted to its adjacent agents. We further denote $\tilde{\theta}_{i}=\theta_{i}\left(t_{k}^{i}\right), \forall t \in\left[t_{k}^{i}, t_{k+1}^{i}\right)$, where $t_{k}^{i}$ denotes $k$ th time at which $i$ th agent communicates with its neighbours. Then, an event-based distributed algorithm for each agent is proposed as

$$
\begin{aligned}
& \dot{P}_{i}=-\nabla C_{i}\left(P_{i}\right)-\varepsilon_{i} \theta_{i} \\
& \dot{\theta}_{i}=-\alpha \sum_{j \in N} a_{i j}\left(\tilde{\theta}_{i}-\tilde{\theta}_{j}\right)-z_{i}+\left(\varepsilon_{i} P_{i}-r_{i} P_{m}\right) \\
& \dot{z}_{i}=\sum_{j \in N} a_{i j}\left(\tilde{\theta}_{i}-\tilde{\theta}_{j}\right)
\end{aligned}
$$

where $\alpha$ is a positive constant to be designed later. Assuming $r_{i}$ is the ability of $i$ th agent to detect the active power mismatch, and we denote $r=\left[r_{1}, \ldots, r_{n}\right]^{T}$ with $\mathbf{1}_{n}^{T} r=1$, for $i \in n$., where $\mathbf{1}_{n}=[1,1, \ldots, 1]^{T} \in \mathbb{R}^{n}$.

The proposed algorithm (16) is distributed with a driving command $-\nabla C_{i}\left(P_{i}\right)-\varepsilon_{i} \theta_{i}$ for $i$ th agent, which is inspired by the central saddle-point dynamics. To observe the global mismatch information, the algorithm (16) takes the advantage of the distributed average estimation method to reconstruct a local copy of saddle-point dynamics scaled by $(1 / N)$. Therefore, the information required to process the algorithm of $i$ th agent is $\tilde{\theta}_{j}$ from its in-neighbours.

\section{A. Convergence Analysis}

For convenience, the algorithm (16) is firstly rewritten as a compact form,

$$
\begin{aligned}
\dot{P} & =-\nabla C(P)-\varepsilon \theta \\
\dot{\theta} & =-\alpha \mathcal{L} \tilde{\theta}-z+\left(\varepsilon P-r P_{m}\right) \\
\dot{z} & =\alpha \mathcal{L} \tilde{\theta}
\end{aligned}
$$

where $P=\left[P_{1}, \ldots, P_{N}\right]^{T}, \theta=\left[\theta_{1}, \ldots, \theta_{N}\right]^{T}, \tilde{\theta}=$ $\left[\tilde{\theta}_{1}, \ldots, \tilde{\theta}_{N}\right]^{T}, \quad \nabla C(P)=\sum_{i \in N} C_{i}\left(P_{i}\right)$ and $z=$ $\left[z_{1}, \ldots, z_{N}\right]^{T}$, respectively, and let $\varepsilon=\operatorname{diag}\left(\varepsilon_{1}, \ldots, \varepsilon_{N}\right)$. 
The convergence analysis starts with characterizing the equilibrium point of (17). It can obtain the equilibrium point of (17) by

$$
\begin{aligned}
& 0=-\nabla C(\bar{P})-\varepsilon \bar{\theta} \\
& 0=-\alpha \mathcal{L} \tilde{\theta}-\bar{z}+\left(\varepsilon \bar{P}-r P_{m}\right) \\
& 0=\alpha \mathcal{L} \tilde{\theta}
\end{aligned}
$$

where $(\bar{P}, \bar{\theta}, \bar{z})$ denotes the equilibrium point of the proposed algorithm.

When implementing the proposed algorithm in a connected communication graph, one has $\mathbf{1}_{N}^{T} \mathcal{L}=0$. Thus, if our algorithm starts from $\mathbf{1}_{n}^{T} z(0)=0$, one has,

$$
\begin{aligned}
\mathbf{1}_{n}^{T} \dot{z}=\mathbf{1}_{n}^{T} \mathcal{L} \tilde{\theta} & =0 \\
\rightarrow \quad \mathbf{1}_{n}^{T} z(t) & =\mathbf{1}_{n}^{T} z(0)=0, \quad \forall t \geq 0 .
\end{aligned}
$$

Then, multiplying (18b) with $\mathbf{1}_{n}^{T}$ results in,

$$
\begin{aligned}
& \sum_{i \in N} \varepsilon_{i} \overline{P_{i}}-P_{m}=0 \\
& \nabla C_{i}\left(\bar{P}_{i}\right)+\varepsilon_{i} \bar{\theta}=0 .
\end{aligned}
$$

By comparing (20) with the KKT condition (13), it shows that the optimization problem is solved if the algorithm (17) converges to its equilibrium set.

For $i$ th agent, a measurement error is defined as

$$
e_{i}(t)=\tilde{\theta}_{i}(t)-\theta_{i}(t), \quad i=1, \ldots, N .
$$

Then, the event condition is given by

$$
f_{i}(t)=4 d_{i}\left\|e_{i}\right\|^{2}-\sum_{j \in N} a_{i j}\left\|\tilde{\theta}_{i}-\tilde{\theta}_{j}\right\|^{2}-\kappa e^{-\mu t}
$$

where $d_{i}$ is the degree of $i$ th agent, and $\kappa, \mu \in \mathbb{R}^{+}$. An event is triggered for $i$ th agent if the event function $f_{i}(t) \geq 0$ is satisfied. Specifically, $i$ th agent will update $\sum_{i \in N} a_{i j}\left(\tilde{\theta}_{i}-\tilde{\theta}_{j}\right)$ using its current state at the instant. Meanwhile, $i$ th agent will also broadcast its current state to its in-neighbours through the communication network, and reset the measurement error $e_{i}$ to zero. Additionally, $i$ th agent will receive the sampled estimation of the supply-demand mismatch from its adjacent agents if an event of any of them is triggered. As a result, using this mechanism will reduce the communication cost.

Remark 4.1: Event-based distributed energy management has been investigated in pioneer work [25]. However, the results in [25] requires the information exchange of two auxiliary variables. By exploring the properties of positive invariant set, algorithm (16) only needs to transmit $\tilde{\theta}_{i}$, which reduces the communication cost. Furthermore, the time-dependent threshold $\kappa e^{-\mu t}$ in the event function (22) brings extra design freedom to achieve the trade-off between the communication cost and the convergence rate. In fact, any positive $\kappa$ and $\mu$ can guarantee the convergence of the system. In addition, the system with a large $\mu$ and a smaller $\kappa$ will have a faster convergence, but more frequent communications. The design of $\kappa$ and $\mu$ relies on the requirement of the convergence rate and the communication cost.

Remark 4.2: Since continuous-time algorithms with periodic communication need consider worst-case situations to determine appropriate periods, they might cause a wasteful use of the communication resources [35]. In the proposed algorithm, each agent only communicates when the event function $f_{i}(t) \geq 0$ so that it could use the communication resources effectively.

Theorem 4.1: Assume that each $C_{i}\left(P_{i}\right)$ is $m^{i}$-strongly convex with $m=\min \left\{m^{1}, \ldots, m^{N}\right\}$. Then, for each $i \in N$ in the connected graph, the proposed algorithm (17) starting from $P_{i}(0), \theta_{i}(0) \in \mathbb{R}$ with $\sum_{i \in N} z_{i}(0)=0$ will converge to the solution set $\left(P^{*}, \theta^{*}\right)$ of the optimization problem (11) if the event function $f_{i} \geq 0$ is fulfilled, and there exists $\alpha$ such that

$$
\alpha \lambda_{2}-(\delta+1)^{2} \geq 0
$$

such that

$$
m(\delta+1)>\left\|\varepsilon^{T} R R^{T} \varepsilon\right\|, \delta \in \mathbb{R}_{>0}
$$

where $\lambda_{2}$ is the smallest non-zero eigenvalue of $\mathcal{L}$.

Proof: Our analysis starts from transferring the equilibrium point of (17) to the origin such as

$$
\begin{gathered}
\hat{P}=P-P^{*}, \quad \hat{\theta}=\Gamma^{T}\left(\theta-\theta^{*} \mathbf{1}_{N}\right), \\
\hat{z}=\Gamma^{T}\left(z-\left(\varepsilon P^{*}-r P_{m}\right)\right)
\end{gathered}
$$

where $\Gamma=[r, R]$ is an orthogonal matrix [35] with

$$
r^{T} R=0, R^{T} R=I_{N-1}, \quad R R^{T}=I_{N}-r r^{T}
$$

where $r=\frac{1}{\sqrt{N}} \mathbf{1}_{N} \in \mathbb{R}$ and $R \in \mathbb{R}^{N \times(N-1)}$. Then, the algorithm (17) reads as

$$
\begin{aligned}
\dot{\hat{P}} & =-y\left(P, P^{*}\right)-\varepsilon \Gamma \hat{\theta} \\
\dot{\hat{\theta}}_{1} & =r^{T} \varepsilon \hat{P} \\
\dot{\hat{\theta}}_{2: N} & =-\alpha R^{T} \mathcal{L} R \tilde{\theta}_{2: N}-\hat{z}_{2: N}+R^{T} \varepsilon \hat{P} \\
\dot{\hat{z}}_{1} & =0 \\
\dot{\hat{z}}_{2: N} & =\alpha R^{T} \mathcal{L} R \tilde{\theta}_{2: N}
\end{aligned}
$$

where $y\left(P, P^{*}\right):=\left(\nabla C\left(\hat{P}+P^{*}\right)-\nabla C\left(P^{*}\right)\right)$. To study the stability of (27), a positive-definite Lyapunov function is considered as

$$
\begin{aligned}
V= & \frac{\delta+1}{2} \hat{P}^{T} \hat{P}+\frac{\delta}{2} \hat{\theta}_{2: N}^{T} \hat{\theta}_{2: N}+\frac{\delta+1}{2} \hat{\theta}_{1}^{T} \hat{\theta}_{1} \\
& +\frac{1}{2}\left(\hat{\theta}_{2: N}+\hat{z}_{2: N}\right)^{T}\left(\hat{\theta}_{2: N}+\hat{z}_{2: N}\right)
\end{aligned}
$$

with $\delta>0$.

The Lie derivative of $V$ along (27) is

$$
\begin{aligned}
\dot{V}= & -(\delta+1) \hat{P}^{T} y\left(P, P^{*}\right)-(\delta+1) \hat{\theta}_{2: N}^{T} \hat{z}_{2: N} \\
& -\hat{z}_{2: N}^{T} \hat{z}_{2: N}+\hat{z}_{2: N}^{T} \varepsilon R^{T} \hat{P}-\alpha \delta \hat{\theta}_{2: N}^{T} R^{T} \mathcal{L} R \tilde{\theta}_{2: N} \\
= & -(\delta+1) \hat{P}^{T} y\left(P, P^{*}\right)-(\delta+1) \hat{\theta}_{2: N}^{T} \hat{z}_{2: N} \\
& -\hat{z}_{2: N}^{T} \hat{z}_{2: N}+\hat{z}_{2: N}^{T} \varepsilon R^{T} \hat{P}-\frac{\delta}{2} \alpha \hat{\theta}_{2: N}^{T} \mathcal{L} \hat{\theta}_{2: N}+\frac{\delta}{2} \alpha W
\end{aligned}
$$

where $W=-\hat{\theta}_{2: N}^{T} R^{T} \mathcal{L} R \hat{\theta}-2 \hat{\theta}_{2: N}^{T} R^{T} \mathcal{L} R e_{2: N}$. Next, one can show that

$$
\begin{aligned}
\dot{V} \leq & -m(\delta+1) \hat{P}^{T} \hat{P}-\frac{\delta \alpha}{2} \lambda_{2} \hat{\theta}_{2: N}^{T} \hat{\theta}_{2: N}-(\delta+1) \hat{\theta}_{2: N}^{T} \hat{z}_{2: N} \\
& -\hat{z}_{2: N}^{T} \hat{z}_{2: N}+\hat{z}_{2: N}^{T} \varepsilon R^{T} \hat{P}_{2: N}+\frac{\delta}{2} \alpha W
\end{aligned}
$$


where the fact for the connected graph

$$
-\frac{\delta}{2} \alpha \hat{\theta}_{2: N}^{T} R^{T} \mathcal{L} R \hat{\theta}_{2: N} \leq-\frac{\delta}{2} \alpha \lambda_{2} \hat{\theta}_{2: N}^{T} \hat{\theta}_{2: N}
$$

and the property of $m$-strong convexity such that

$$
-\hat{P}^{T}\left(\nabla C\left(P+P^{*}\right)-\nabla\left(P^{*}\right)\right) \leq-m \hat{P}^{T} \hat{P}
$$

are applied to deduce the inequality of $\dot{V}$. Next, the Young's inequality is further adopted such that

$$
\begin{array}{r}
-(\delta+1) \hat{\theta}_{2: N}^{T} \hat{z}_{2: N} \leq \frac{(\delta+1)^{2}}{2} \hat{\theta}_{2: N}^{T} \hat{\theta}_{2: N}+\frac{1}{2} \hat{z}_{2: N}^{T} \hat{z}_{2: N} \\
\hat{z}_{2: N}^{T} \varepsilon R^{T} \hat{P} \leq \frac{1}{4} \hat{z}_{2: N}^{T} \hat{z}_{2: N}+\hat{P}^{T}\left(\varepsilon^{T} R R^{T} \varepsilon\right) \hat{P}
\end{array}
$$

to establish an upper bound of $\dot{V}$ such as

$$
\begin{aligned}
\dot{V} \leq & -\hat{P}^{T}\left(m(\delta+1) I-\varepsilon^{T} R R^{T} \varepsilon\right) \hat{P}-\frac{1}{4} \hat{z}_{2: N}^{T} \hat{z}_{2: N} \\
& -\left(\frac{\delta \alpha}{2} \lambda_{2}-\frac{(\delta+1)^{2}}{2}\right) \hat{\theta}_{2: N}^{T} \hat{\theta}_{2: N}+\frac{\delta}{2} \alpha W \\
= & -\bar{S}+\frac{\delta}{2} \alpha W
\end{aligned}
$$

where, by the virtue of (23) and (24),

$$
\begin{aligned}
\bar{S}= & \hat{P}^{T}\left(m(\delta+1) \mathbf{I}-\varepsilon^{T} R R^{T} \varepsilon\right) \hat{P}+\frac{1}{4} \hat{z}_{2: N}^{T} \hat{z}_{2: N} \\
& +\left(\frac{\delta \alpha}{2} \lambda_{2}-\frac{(\delta+1)^{2}}{2}\right) \hat{\theta}_{2: N}^{T} \hat{\theta}_{2: N} \geq 0 .
\end{aligned}
$$

Next, using $R^{T} R=I_{N}-\frac{1}{N} 1_{N} 1_{N}^{T}$ and $\left(I_{N}-\frac{1}{N} 1_{N} 1_{N}^{T}\right) \mathcal{L}=$ $\mathcal{L}\left(I_{N}-\frac{1}{N} 1_{N} 1_{N}^{T}\right)$, we can obtain that

$$
\hat{\theta}_{2: N}=R^{T} \theta, \quad \tilde{\theta}_{2: N}=R^{T} \tilde{\theta} .
$$

Then, one has

$$
\begin{aligned}
W & =-(\tilde{\theta}-e)^{T} \mathcal{L}(\tilde{\theta}-e)-2(\tilde{\theta}-e)^{T} \mathcal{L} e \\
& =e^{T} \mathcal{L} e-\tilde{\theta}^{T} \mathcal{L} \tilde{\theta}
\end{aligned}
$$

Since $a_{i j}=a_{j i}$, it can be obtained that

$$
\begin{aligned}
e^{T} \mathcal{L} e & =\sum_{i=1}^{N} \sum_{j=1}^{N} a_{i j} e_{i}^{T}\left(e_{i}-e_{j}\right) \\
& \leq \frac{3}{2} \sum_{i=1}^{N} \sum_{j=1}^{N} a_{i j} e_{i}^{T} e_{i}+\frac{1}{2} \sum_{i=1}^{N} \sum_{j=1}^{N} a_{i j} e_{j}^{T} e_{j} \\
& =2 \sum_{i=1}^{N} d_{i}\left\|e_{i}\right\|^{2}
\end{aligned}
$$

and

$$
\tilde{\theta}^{T} \mathcal{L} \tilde{\theta}=\frac{1}{2} \sum_{i=1}^{N} \sum_{j=1}^{N} a_{i j}\left\|\tilde{\theta}_{i}-\tilde{\theta}_{j}\right\|^{2} .
$$

Thus, we have

$$
W=\frac{1}{2} \sum_{i=1}^{N}\left(4 \sum_{i=1}^{N} d_{i}\left\|e_{i}\right\|^{2}-\sum_{j=1}^{N} a_{i j}\left\|\tilde{\theta}_{i}-\tilde{\theta}_{j}\right\|^{2}\right) .
$$

With (22), it yields

$$
\begin{aligned}
\dot{V} & \leq-\bar{S}+\frac{\delta \alpha}{4} \sum_{i=1}^{N}\left(4 \sum_{i=1}^{N} d_{i}\left\|e_{i}\right\|^{2}-\sum_{j=1}^{N} a_{i j}\left\|\tilde{\theta}_{i}-\tilde{\theta}_{j}\right\|^{2}\right) \\
& \leq-\bar{S}+\frac{N \kappa \delta \alpha}{4} e^{-\mu t} .
\end{aligned}
$$

Then, we can further rewrite (42) as

$$
\begin{aligned}
\dot{V} & =-h_{2}^{T} F h_{2}+\frac{N \kappa \delta \alpha}{4} e^{-\mu t} \\
& \leq-\lambda_{\min }(F) h_{2}^{T} h_{2}+\frac{N \kappa \delta \alpha}{4} e^{-\mu t}
\end{aligned}
$$

where $h_{2}=\left[\hat{P}, \hat{\theta}_{2: N}, \hat{z}_{2: N}\right]$ and $F=\operatorname{diag}\left(\gamma_{1}, \gamma_{2}, \gamma_{3}\right)$ with $\gamma_{1}=m(\delta+1) I-\varepsilon^{T} R R^{T} \varepsilon, \gamma_{2}=-\left(\frac{\delta \alpha}{2} \lambda_{2}-\frac{(\delta+1)^{2}}{2}\right) I_{N}$ and $\gamma_{3}=\frac{1}{4} I_{N}$. Then, one has

$$
0 \leq V(t) \leq \frac{N \kappa \delta \alpha}{4} \int_{0}^{t} e^{-\mu \tau} d \tau
$$

which shows $V$ is bounded. Then $h_{2}$ is bounded according to (28), which further implies that $\dot{h}_{2}$ is bounded by invoking (27). In addition, from (43), it can obtain that

$V(\infty)-V(0) \leq-\int_{0}^{\infty}-\lambda_{\min }(F) h_{2}(\tau)^{T} h_{2}(\tau) d \tau+\frac{N \kappa \delta \alpha}{4 \mu}$,

which can rewritten as

$$
\int_{0}^{\infty} h_{2}(\tau)^{T} h_{2}(\tau) d \tau \leq \frac{1}{\lambda_{\min }(F)}\left[V(\infty)-V(0)+\frac{N \kappa \delta \alpha}{4 \mu}\right] .
$$

As a result, with the Barbalat's Lemma [36], we can conclude that $h_{2} \rightarrow 0$ as $t \rightarrow \infty$, which indicates that the trajectories of (27) converge to the solution of the KKT equation given in (13).

Next, the final objective is to show our algorithm does not exhibit the Zeno behaviour, which can follow a similar line in [37]. Due to the page limit, we omit it.

\section{Simulation Studies}

In this section, three case studies are presented to validate the effectiveness of the event-based optimization algorithm, where $\kappa=2.5$ and $\mu=0.8$. The battery cell cost is 0.058 $\$ / \mathrm{kW}$. In the first two cases, the proposed distributed algorithm is tested in a modified IEEE 14-bus system as depicted in Fig. 2, including two CGs, three BESSs and one WT, which is widely adopted in energy management problem [16], [38], [39] . The simulation parameters are summarized in Table. I where the power loss coefficients are adopted in [40], and the topology of the communication network is assumed to be identical to the physical network.

In Case 5.1, the performance of the proposed algorithm is investigated under the constant renewable generation and load demands. Case 5.2 is carried out with varying output power from the renewable generation. Finally, the scalability of the proposed algorithm is investigated in Case 5.3 with the IEEE 162-bus system. 


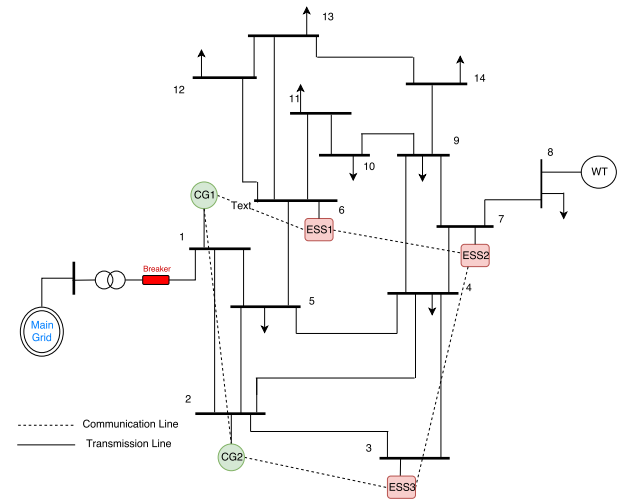

Fig. 2. IEEE 14-bus system with five BESSs

TABLE I

PARAMETERS OF SIMULATION STUDIES

\begin{tabular}{llllll}
\hline & $\alpha_{i}$ & $\beta_{i}$ & $P_{i}^{\min }(\mathrm{kW})$ & $P_{i}^{\max }(\mathrm{kW})$ & $\eta_{i}$ \\
\hline CG1 & 0.086 & 2.482 & 20 & 70 & 0.042 \\
CG2 & 0.093 & 2.688 & 25 & 65 & 0.065 \\
BESS1 & 0.489 & 0.081 & -20 & 45 & 0.051 \\
BESS2 & 0.2092 & 0.072 & -20 & 50 & 0.040 \\
BESS3 & 0.2247 & 0.061 & -15 & 45 & 0.063 \\
\hline
\end{tabular}

\section{A. Case 1: Constant Renewable Generation}

In this case, the proposed distributed solution is tested in the IEEE 14-bus system under a constant supply-demand mismatch as $200 \mathrm{~kW}$. The microgrid is assumed to be operated in the islanded mode, and the topology of the communication network is given by Fig. 3. BESS4 and BESS5 are assumed to be charged at the beginning. As shown in Fig. 4, the marginal cost of each participant converges to its optimal value firstly. The updates of the power generation, the estimation of the supply-demand mismatch, and the triggering instants are depicted in Figs. 5 - 7. As shown in the results, the power reference of each agent converges to its optimal value in response to the marginal cost update and meanwhile, the supply-demand mismatch converges to zero by the proposed algorithm. Besides, as indicated in Figs. 7, the total update number of the algorithm is dramatically reduced to achieve the optimal value. To further illustrate the effectiveness of our solution, the existing work [13] with the periodic communication is adopted to compare the performance. In this case, the periodic interval time is set as $0.02 \mathrm{~s}$ and the convergence coefficient is 0.0005 provided by [13]. The results are given in Table. II where the number of communications of 2 nd agent is taken as an example. It can verify that the proposed eventbased algorithm require less information exchanges among agents in the network compared with periodic communication scheme ( $10.2 \%$ of the instants by the periodical one).

TABLE II

THE TIME INSTANTS OF 2TH AGENT UNDER DIFFERENT ALGORITHMS

\begin{tabular}{lcc}
\hline & Event-based & Periodic [13] \\
\hline Instants & 486 & 4764 \\
\hline
\end{tabular}

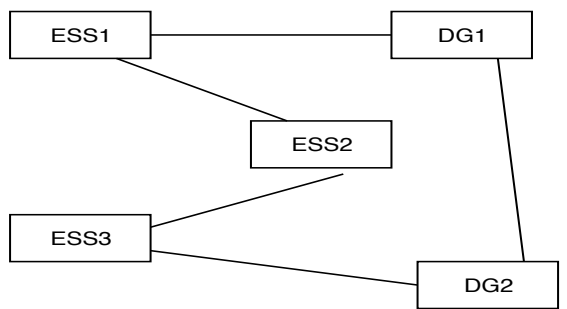

Fig. 3. The topology of the communication network

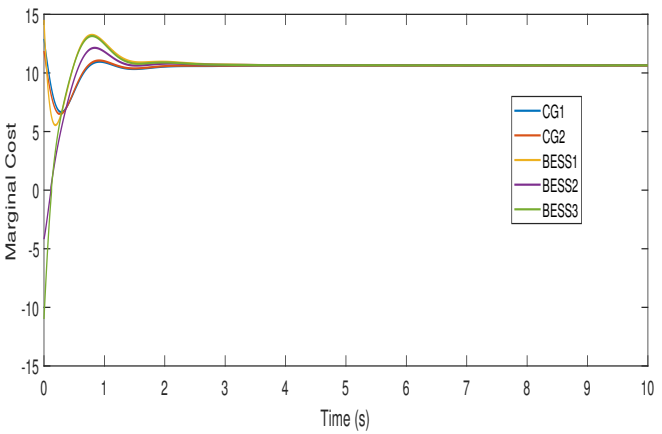

Fig. 4. Marginal cost updates under constant mismatches

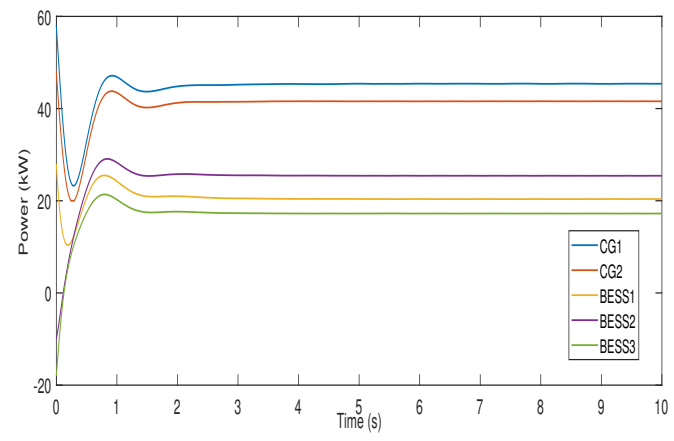

Fig. 5. Local generation updates under constant mismatches

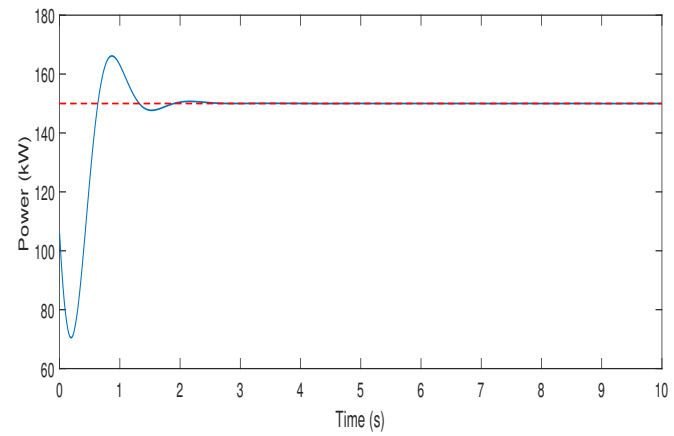

Fig. 6. The estimation of the supply-demand mismatch 


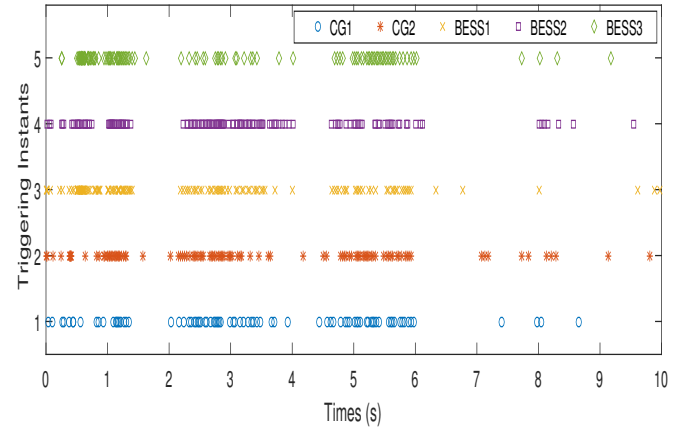

Fig. 7. The triggering instants of each agent under constant mismatches

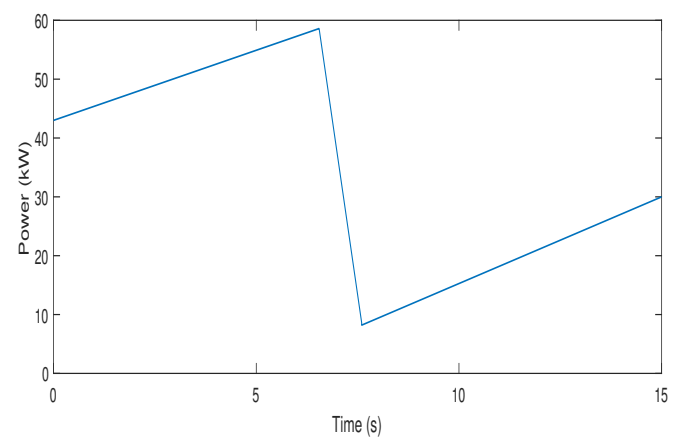

Fig. 8. The output power from the wind turbine

\section{B. Case 2: Varying Renewable Generation}

Since the output power from RGs would be uncertain and unpredictable, the required power from CGs and BESSs will be time-varying when high intermittent RGs are integrated into the microgrid. In this case, the reactive power regulation mode is adopted to control the WT and the output power from the WT is given by Fig. 8, which shows the WT generation suddenly drops to a very low value within $1 \mathrm{~s}$. The microgrid is operated under islanded mode in $0 \mathrm{~s}$ intentionally by controlling the breaker. Figs. 9 - 11 present the results for the update of the marginal cost, the power generation, and the power balance estimation, respectively. The results show that the marginal cost converges to the optimal value according to the current generation from the WT. Accordingly, the power outputs from dispatchable units converge to the corresponding values to diminish the mismatch caused by the varying renewable generation. The frequency response given in Fig. 13 shows the system frequency is restored to its nominal value, i.e., $50 \mathrm{~Hz}$, when the mismatch is diminished. Also, the time instant of each agent is shown in Fig. 12, which shows the communication requirements are reduced effectively even under the varying mismatch.

\section{Case 3: Scalability Analysis}

Considering a future smart grid could be a large-scale system, the scalability of the proposed algorithm is investigated in this case under the IEEE 162-bus system with eight CGs and nine BESSs where the simulation parameters are adopted from [41] and the total load demand is given by 18422 MW. Deploying the proposed algorithm, as shown in Fig. 14,

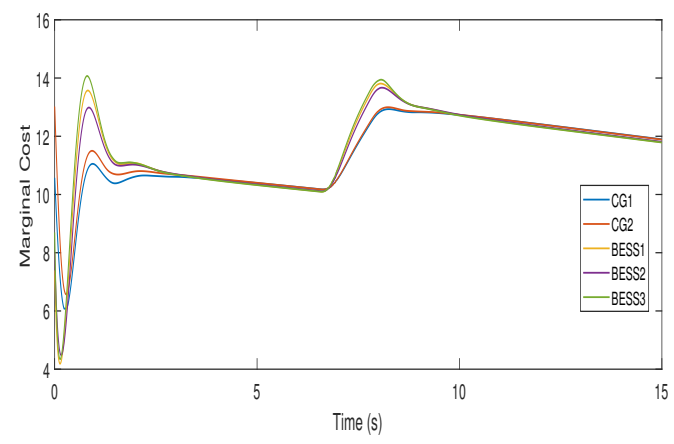

Fig. 9. Marginal cost updates under varying mismatches

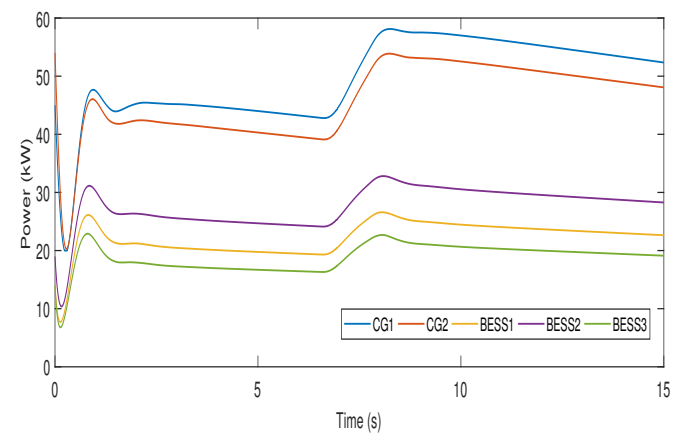

Fig. 10. Local generation updates under varying mismatches

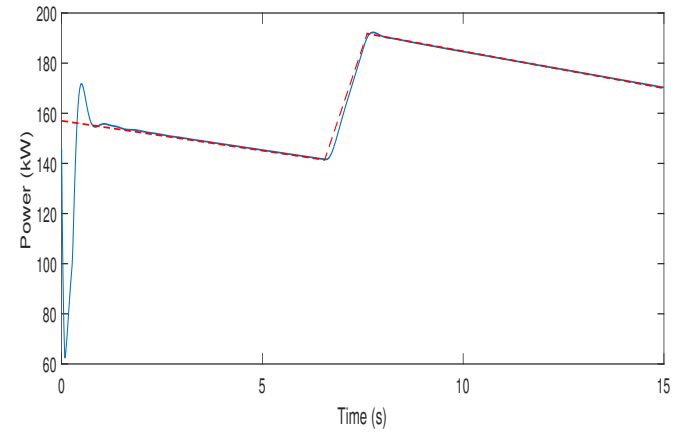

Fig. 11. The estimation of the supply-demand mismatch

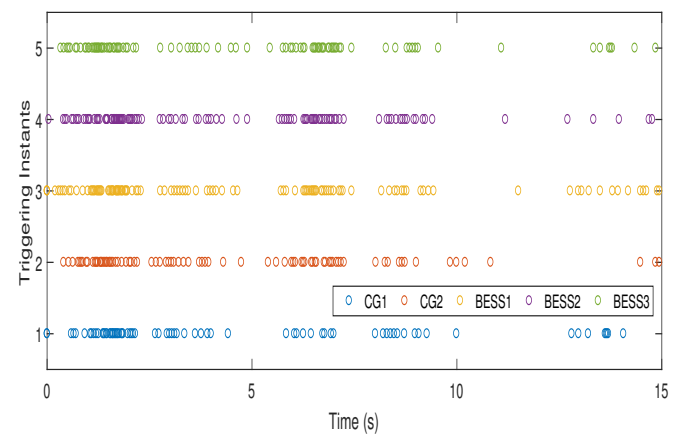

Fig. 12. The triggering instants of each agent under varying mismatches 


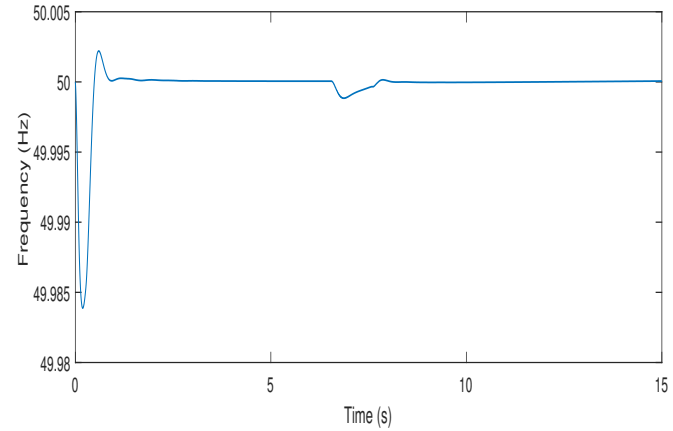

Fig. 13. The frequency response under varying renewable generation

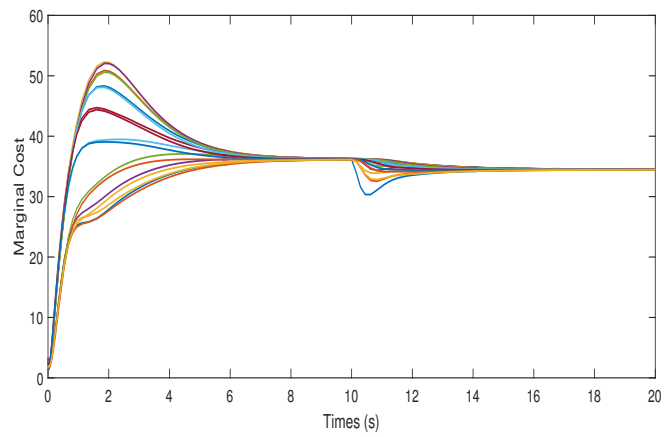

Fig. 14. Marginal cost updates

the marginal cost will converge to a common value, which indicates the effectiveness of our algorithm will not be affected by the increasing in the system scale.

\section{CONCLuSion}

In this paper, an event-based distributed optimization algorithm is proposed for the optimal energy management in a microgrid. The proposed solution can minimize the generation cost of energy suppliers effectively considering the supplydemand balance within the microgrid. Based on the MAS framework, our algorithm can be implemented in a distributed manner without the central communication. Besides, an eventtriggered mechanism is adopted to reduce the requirements of the agent communication. Simulation results in both the IEEE 14-bus and 162-bus systems have been given to verify the effectiveness of the proposed algorithm.

\section{APPENDIX}

\section{A. The Convergence Analysis of The Distributed Algorithm With Inequality Constraints}

Using differential inclusions and the non-smooth analysis [17], for $P_{i}(0) \in \mathcal{P}_{i}$, an event-based algorithm with inequality constraints is designed as,

$$
\begin{aligned}
& \dot{P}_{i} \in \Pi_{\mathcal{P}_{i}}\left(P_{i},-\nabla C_{i}\left(P_{i}\right)-\varepsilon_{i} \theta_{i}\right) \\
& \dot{\theta}_{i}=-\alpha \sum_{j \in N} a_{i j}\left(\tilde{\theta}_{i}-\tilde{\theta}_{j}\right)-z_{i}+\left(\varepsilon_{i} P_{i}-r_{i} P_{m}\right) \\
& \dot{z}_{i}=\sum_{j \in N} a_{i j}\left(\tilde{\theta}_{i}-\tilde{\theta}_{j}\right)
\end{aligned}
$$

where $\alpha$ is a positive constant. The algorithms (47) and (16) share the same structure, where (47) includes a differential inclusion and a differentiated projection operation to deal with inequality constraints. Before we presenting the convergence proof, several following lemmas are useful for our analysis.

Lemma A.1: [42] Let $\mathcal{F}: \mathbb{R}^{n} \rightrightarrows \mathbb{R}^{n}$ be a set-valued map and $\mathcal{X}$ denote a convex set. For $x(0)=x_{0}$, then consider,

$$
\begin{aligned}
& \dot{x} \in \mathcal{F}(x)-\mathcal{N}_{\mathcal{X}}(x) \\
& \dot{x} \in \Pi_{\mathcal{X}}(x, \mathcal{F}(x)) .
\end{aligned}
$$

where $\mathcal{N}_{\mathcal{X}}(x)$ is the normal cone of set $\mathcal{X}$ at $x$. Thus, the following statements hold:

1) A trajectory $x(\cdot)$ is a solution to (48) if and only if it is a solution to (49).

2) If $\mathcal{F}$ is bounded over $\mathcal{X}$, then there exists a solution to (48) and (49)

Following [43], it can obtain the optimal condition for the problem (11).

Lemma A.2: If $P_{i}^{*}, i \in 1, \ldots, N$ is the minimum of the problem (11), there exists $\theta^{*} \in \mathbb{R}^{n}$ such that

$$
0_{n} \in \partial C_{i}\left(P_{i}^{*}\right) \varepsilon_{i}+\mathcal{N}_{\mathcal{P}_{i}}\left(P_{i}^{*}\right)
$$

Conversely, if (50) holds for a feasible point $P_{i}^{*}, i \in 1, \ldots, N$, and $\theta^{*} \in \mathbb{R}^{n}$, and thus, $P_{i}^{*}, i \in 1, \ldots, N$ is the global minimum of the problem (11).

Again, we write (47) as a compact form with $P(0) \in \mathcal{P}$,

$$
\begin{aligned}
\dot{P} & \in \Pi_{\mathcal{P}}(P,-\nabla C(P)-\varepsilon \theta) \\
\dot{\theta} & =-\alpha \mathcal{L} \tilde{\theta}-z+\left(\varepsilon P-r P_{m}\right) \\
\dot{z} & =\alpha \mathcal{L} \tilde{\theta}
\end{aligned}
$$

Using Lemma A.1, the solution existence of (51) is guaranteed. Following the analysis of Lemma 5 in [44], the problem (11) is solved by a solution, $\bar{P}$, if $(\bar{P}, \bar{\theta}, \bar{z})$ is the equilibrium point of (51).

Proof: The proof starts from transforming the equilibrium point to the origin. It follows [45] that,

$$
\Pi_{\mathcal{P}_{i}}\left(P_{i},-\nabla C_{i}\left(P_{i}\right)-\varepsilon_{i} \theta_{i}\right) \subset-\nabla C_{i}\left(P_{i}\right)-\varepsilon_{i} \theta_{i}-\mathcal{N}_{\mathcal{P}_{i}}\left(P_{i}\right),
$$

and thus $-\nabla C_{i}\left(P_{i}^{*}\right)-\varepsilon_{i} \theta_{i}^{*} \subset \mathcal{N}_{\mathcal{P}_{i}}\left(P_{i}^{*}\right)$. Following a similar coordinate transformation in the proof of Theorem 4.1, one has

$$
\begin{gathered}
\left\{\sigma \in \mathbb{R}: \sigma=-w\left(P, P^{*}\right)-\varepsilon \Gamma \hat{\theta}\right. \\
\left.w\left(P, P^{*}\right) \in \nabla C(P)-\nabla C\left(P^{*}\right)+\mathcal{N}_{\mathcal{P}}(P)-\mathcal{N}_{\mathcal{P}}\left(P^{*}\right)\right\}
\end{gathered}
$$

$$
\begin{aligned}
\dot{\hat{\theta}}_{1} & =r^{T} \varepsilon \hat{P} \\
\dot{\hat{\theta}}_{2: N} & =-\alpha R^{T} \mathcal{L} R \tilde{\theta}_{2: N}-\hat{z}_{2: N}+R^{T} \varepsilon \hat{P} \\
\dot{\hat{z}}_{1} & =0 \\
\dot{\hat{z}}_{2: N} & =\alpha R^{T} \mathcal{L} R \tilde{\theta}_{2: N} .
\end{aligned}
$$


Next, we sketch the convergence analysis of (53) by adopting the candidate Lyapunov function $V$. Its set-valued Lie derivative along (53) is

$$
\begin{aligned}
\mathcal{L}_{(53)} V= & \left\{\sigma \in \mathbb{R}: \sigma=-(\delta+1) \hat{P}^{T} w\left(P, P^{*}\right)\right. \\
& -(\delta+1) \hat{\theta}_{2: N}^{T} \hat{z}_{2: N}-\hat{z}_{2: N}^{T} \hat{z}_{2: N}+\hat{z}_{2: N}^{T} \varepsilon R^{T} \hat{P} \\
& -\frac{\delta}{2} \alpha \hat{\theta}_{2: N}^{T} \mathcal{L} \hat{\theta}_{2: N}+\frac{\delta}{2} \alpha W \\
w\left(P, P^{*}\right) & \left.\in \nabla C(P)-\nabla C\left(P^{*}\right)+\mathcal{N}_{\mathcal{P}}(P)-\mathcal{N}_{\mathcal{P}}\left(P^{*}\right)\right\}
\end{aligned}
$$

where $W=-\hat{\theta}_{2: N}^{T} R^{T} \mathcal{L} R \hat{\theta}-2 \hat{\theta}_{2: N}^{T} R^{T} \mathcal{L} R e_{2: N}$. Note that $\left\langle P_{i}-P_{i}^{*}, \mathcal{N}_{\mathcal{P}_{i}}\left(P_{i}\right)\right\rangle \subseteq \mathbb{R}_{+}$and $\left\langle P_{i}-P_{i}^{*}, \mathcal{N}_{\mathcal{P}_{i}}\left(P_{i}^{*}\right)\right\rangle \subseteq$ $\mathbb{R}_{-}$, there is $\left\langle\hat{P}, \mathcal{N}_{\mathcal{P}}(P)-\mathcal{N}_{\mathcal{P}}\left(P^{*}\right)\right\rangle \subseteq \mathbb{R}_{+}$. Hence, $-\hat{P}^{T} w\left(P, P^{*}\right) \in-\left\langle\hat{P}, \partial C(P)-\partial C\left(P^{*}\right)\right\rangle-\left\langle\hat{P}, \mathcal{N}_{\mathcal{P}}(P)-\right.$ $\left.\mathcal{N}_{\mathcal{P}}\left(P^{*}\right)\right\rangle$. Therefore, using the property of $m$-strong convexity of $C(\cdot)$, we have

$$
\begin{aligned}
\max & \left\{\sigma \in \mathbb{R}: \sigma=-(\delta+1) \hat{P}^{T} w\left(P, P^{*}\right)\right. \\
& \left.w\left(P, P^{*}\right) \in \nabla C(P)-\nabla C\left(P^{*}\right)+\mathcal{N}_{\mathcal{P}}(P)-\mathcal{N}_{\mathcal{P}}(P)\right\} \\
& \leq-(\delta+1) m \hat{P}^{T} \hat{P} .
\end{aligned}
$$

Then we can follow the similar lines in the proof of Theorem 4.1, and one has,

$$
\begin{aligned}
\max \mathcal{L}_{(53)} V & \leq-\bar{S}+\frac{N \kappa \delta \alpha}{4} e^{-\mu t} \\
& \leq-\lambda_{\min }(F) h_{2}^{T} h_{2}+\frac{N \kappa \delta \alpha}{4} e^{-\mu t}
\end{aligned}
$$

where $\bar{S} \geq 0$ given by (36). Integrating (56), we further have,

$$
0 \leq V(t) \leq \frac{N \kappa \delta \alpha}{4} \int_{0}^{t} e^{-\mu \tau} d \tau .
$$

Therefore, following [46], $V$ is lower bounded. Then, it can conclude a similar result given by (45) and (46), which indicates the trajectories of (53) converge to the solution of the problem (11).

\section{REFERENCES}

[1] H. K. Nunna and S. Doolla, "Multiagent-based distributed-energyresource management for intelligent microgrids," IEEE Trans. Ind. Electron., vol. 60, no. 4, pp. 1678-1687, 2013.

[2] Q. Jiang, M. Xue, and G. Geng, "Energy management of microgrid in grid-connected and stand-alone modes," IEEE Trans. Power Syst., vol. 28, no. 3, pp. 3380-3389, 2013.

[3] Q. Shafiee, C. Stefanovic, T. Dragicevic, P. Popovski, J. C. Vasquez, and J. M. Guerrero, "Robust networked control scheme for distributed secondary control of islanded microgrids," IEEE Trans. Ind. Electron. vol. 61, no. 10, pp. 5363-5374, 2014.

[4] S. Salinas, M. Li, P. Li, and Y. Fu, "Dynamic energy management for the smart grid with distributed energy resources," IEEE Trans. Smart Grid, vol. 4, no. 4, pp. 2139-2151, 2013.

[5] J.-Y. Fan and L. Zhang, "Real-time economic dispatch with line flow and emission constraints using quadratic programming," IEEE Trans. Power Syst., vol. 13, no. 2, pp. 320-325, 1998.

[6] T. Fang and R. Lahdelma, "Optimization of combined heat and power production with heat storage based on sliding time window method," Applied Energy, vol. 162, pp. 723-732, 2016.

[7] B. Ramachandran, S. K. Srivastava, C. S. Edrington, and D. A. Cartes, "An intelligent auction scheme for smart grid market using a hybrid immune algorithm," IEEE Trans. Ind. Electron., vol. 58, no. 10, pp. 4603-4612, 2011.

[8] K. T. Chaturvedi, M. Pandit, and L. Srivastava, "Self-organizing hierarchical particle swarm optimization for nonconvex economic dispatch," IEEE Trans. Power Syst., vol. 23, no. 3, pp. 1079-1087, 2008.
[9] H. Xin, Z. Qu, J. Seuss, and A. Maknouninejad, "A self-organizing strategy for power flow control of photovoltaic generators in a distribution network," IEEE Trans. Power Syst., vol. 26, no. 3, pp. 1462-1473, 2011.

[10] D. Papadaskalopoulos and G. Strbac, "Nonlinear and randomized pricing for distributed management of flexible loads," IEEE Trans. Smart Grid, vol. 7, no. 2, pp. 1137-1146, 2016.

[11] N. Rahbari-Asr, U. Ojha, Z. Zhang, and M.-Y. Chow, "Incremental welfare consensus algorithm for cooperative distributed generation/demand response in smart grid," IEEE Trans. Smart Grid, vol. 5, no. 6, pp. 2836-2845, 2014

[12] N. Rahbari-Asr, M. Y. Chow, J. Chen, and R. Deng, "Distributed realtime pricing control for large-scale unidirectional v2g with multiple energy suppliers," IEEE Trans. Ind. Informat., vol. 12, no. 5, pp. $1953-$ 1962, Oct 2016

[13] Z. Zhang and M.-Y. Chow, "Convergence analysis of the incremental cost consensus algorithm under different communication network topologies in a smart grid," IEEE Trans. Power Syst., vol. 27, no. 4, pp. 1761-1768, 2012.

[14] J.-H. Teng, S.-W. Luan, D.-J. Lee, and Y.-Q. Huang, "Optimal charging/discharging scheduling of battery storage systems for distribution systems interconnected with sizeable pv generation systems," IEEE Trans. Power Syst., vol. 28, no. 2, pp. 1425-1433, 2013.

[15] S. Yang, S. Tan, and J.-X. Xu, "Consensus based approach for economic dispatch problem in a smart grid," IEEE Trans. Power Syst., vol. 28, no. 4, pp. 4416-4426, 2013.

[16] Y. Xu and Z. Li, "Distributed optimal resource management based on the consensus algorithm in a microgrid," IEEE Trans. Ind. Electron., vol. 62, no. 4, pp. 2584-2592, 2015.

[17] T. Zhao and Z. Ding, "Distributed initialization-free cost-optimal charging control of plug-in electric vehicles for demand management," IEEE Transactions on Industrial Informatics, vol. 13, no. 6, pp. 2791-2801, Dec 2017.

[18] F. Guo, C. Wen, J. Mao, and Y. D. Song, "Distributed economic dispatch for smart grids with random wind power," IEEE Transactions on Smart Grid, vol. 7, no. 3, pp. 1572-1583, May 2016.

[19] H. Zhang, Y. Li, D. W. Gao, and J. Zhou, "Distributed optimal energy management for energy internet," IEEE Transactions on Industrial Informatics, vol. 13, no. 6, pp. 3081-3097, Dec 2017.

[20] N. Liu, J. Chen, L. Zhu, J. Zhang, and Y. He, "A key management scheme for secure communications of advanced metering infrastructure in smart grid," IEEE Trans. Ind. Electron., vol. 60, no. 10, pp. 47464756, 2013.

[21] V. C. Gungor, D. Sahin, T. Kocak, S. Ergut, C. Buccella, C. Cecati, and G. P. Hancke, "Smart grid technologies: Communication technologies and standards," IEEE Trans. Ind. Informat., vol. 7, no. 4, pp. 529-539, 2011.

[22] P. Wan and M. D. Lemmon, "Event-triggered distributed optimization in sensor networks," in Proceedings of the 2009 International Conference on Information Processing in Sensor Networks. IEEE Computer Society, 2009, pp. 49-60.

[23] X. Meng and T. Chen, "Event based agreement protocols for multi-agent networks," Automatica, vol. 49, no. 7, pp. 2125-2132, 2013.

[24] C. Li, X. Yu, W. Yu, T. Huang, and Z.-W. Liu, "Distributed eventtriggered scheme for economic dispatch in smart grids," IEEE Transactions on Industrial informatics, vol. 12, no. 5, pp. 1775-1785, 2016.

[25] Y. Li, H. Zhang, X. Liang, and B. Huang, "Event-triggered based distributed cooperative energy management for multi-energy systems," IEEE Trans. Ind. Informat, 2018.

[26] M. Erol-Kantarci and H. T. Mouftah, "Energy-efficient information and communication infrastructures in the smart grid: A survey on interactions and open issues," IEEE Communications Surveys Tutorials, vol. 17, no. 1, pp. 179-197, Firstquarter 2015.

[27] Y. Xu, W. Zhang, W. Liu, X. Wang, F. Ferrese, C. Zang, and H. Yu, "Distributed subgradient-based coordination of multiple renewable generators in a microgrid," IEEE Trans. Power Syst., vol. 29, no. 1, pp. 23-33, 2014.

[28] P. Samadi, H. Mohsenian-Rad, R. Schober, and V. W. Wong, "Advanced demand side management for the future smart grid using mechanism design," IEEE Trans. Smart Grid, vol. 3, no. 3, pp. 1170-1180, 2012.

[29] N. Rahbari-Asr, M.-Y. Chow, J. Chen, and R. Deng, "Distributed realtime pricing control for large-scale unidirectional v2 $\mathrm{g}$ with multiple energy suppliers," IEEE Trans. Ind. Informat., vol. 12, no. 5, pp. 19531962, 2016.

[30] W. Zhang, Y. Xu, W. Liu, C. Zang, and H. Yu, "Distributed online optimal energy management for smart grids," IEEE Trans. Ind. Informat., vol. 11 , no. 3 , pp. 717-727, 2015. 
[31] P. Samadi, A.-H. Mohsenian-Rad, R. Schober, V. W. Wong, and J. Jatskevich, "Optimal real-time pricing algorithm based on utility maximization for smart grid," in Smart Grid Communications (SmartGridComm), 2010 First IEEE International Conference on. IEEE, 2010, pp. 415-420.

[32] Y. Xu, W. Zhang, and W. Liu, "Distributed dynamic programmingbased approach for economic dispatch in smart grids," IEEE Trans. Ind. Informat., vol. 11, no. 1, pp. 166-175, 2015.

[33] Y. Xu, W. Zhang, G. Hug, S. Kar, and Z. Li, "Cooperative control of distributed energy storage systems in a microgrid," IEEE Trans. smart grid, vol. 6, no. 1, pp. 238-248, 2015.

[34] B. Zhou, X. Liu, Y. Cao, C. Li, C. Y. Chung, and K. W. Chan, "Optimal scheduling of virtual power plant with battery degradation cost," IET Generation, Transmission \& Distribution, vol. 10, no. 3, pp. 712-725, 2016.

[35] S. S. Kia, J. Cortés, and S. Martínez, "Distributed convex optimization via continuous-time coordination algorithms with discrete-time communication," Automatica, vol. 55, pp. 254-264, 2015.

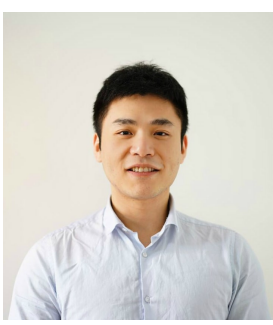

Tianqiao Zhao received his B.Eng. degree in automatic control from North China Electric Power University, Hebei, China, in 2013, and his M.Sc degree in electrical and electronic engineering from the University of Manchester, U.K., in 2014. He is now a $\mathrm{Ph} . \mathrm{D}$. candidate in control engineering with the School of Electrical and Electronic Engineering at the University of Manchester, U.K. His research interests include distributed optimization of microgrids, distributed control and energy storage systems.

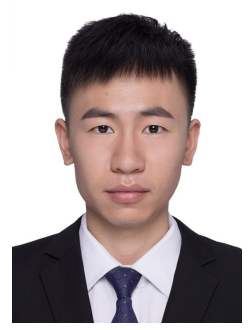

Zhenhong Li received his B.Eng. degree in electrical engineering from Huazhong University of Science and Technology, Hubei, China, in 2013, and the M.Sc. degree in control systems from the University of Manchester, Manchester, U.K., in 2014. He is now a Ph.D. candidate in control engineering with the School of Electrical and Electronic Engineering at the University of Manchester, U.K. His research interests include distributed optimization, and cooperative control of multi-agent systems.
[36] Z. Ding, Nonlinear and Adaptive Control Systems. IET, 2013.

[37] B. Cheng and Z. Li, "Consensus of linear multi-agent systems via fully distributed event-triggered protocols," in Control Conference (CCC), 2017 36th Chinese. IEEE, 2017, pp. 8607-8612.

[38] T. Zhao and Z. Ding, "Distributed agent consensus-based optimal resource management for microgrids," IEEE Trans. Sustainable Energy, vol. 9, no. 1, pp. 443-452, Jan 2018.

[39] Y. F. Eddy, H. B. Gooi, and S. X. Chen, "Multi-agent system for distributed management of microgrids," IEEE Trans. Power Syst., vol. 30, no. 1 , pp. 24-34, 2015 .

[40] D. H. Nguyen, T. Narikiyo, and M. Kawanishi, "Optimal demand response and real-time pricing by a sequential distributed consensusbased admm approach," IEEE Trans. Smart Grid, 2017.

[41] P. S. T. C. Archive:, "162-bus, 17 generator dynamic test case," http: //www.ee.washington.edu/research/pstca/dyn17/pg_tcadd17.htm.

[42] J. Aubin and A. Cellina, "Differential inclusions. 1984."

[43] D. P. Bertsekas, Nonlinear programming. Athena scientific Belmont, 1999.

[44] Z. Deng, S. Liang, and Y. Hong, "Distributed continuous-time algorithms for resource allocation problems over weight-balanced digraphs," IEEE Transactions on Cybernetics, pp. 1-10, 2018.

[45] B. Brogliato, A. Daniilidis, C. Lemarechal, and V. Acary, "On the equivalence between complementarity systems, projected systems and differential inclusions," Systems \& Control Letters, vol. 55, no. 1, pp. 45-51, 2006.

[46] J. Cortes, "Discontinuous dynamical systems," IEEE control Systems, vol. 28 , no. 3, 2008.

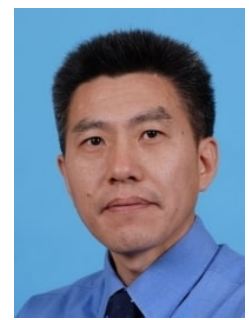

Zhengtao Ding received the B.Eng. degree from Tsinghua University, Beijing, China, and the M.Sc. degree in systems and control and the Ph.D. degree in control systems from the University of Manchester Institute of Science and Technology, Manchester, U.K. After working as a Lecturer with Ngee Ann Polytechnic, Singapore, for ten years, in 2003, he joined The University of Manchester, Manchester, U.K., where he is currently Professor of Control Systems with the School of Electrical and Electronic Engineering. He is the author of the book Nonlinear and Adaptive Control Systems (IET, 2013) and a number of journal papers. His research interests include nonlinear and adaptive control theory and their applications. Prof. Ding serves as an Associate Editor for the IEEE Transactions on AUtomatic Control, IEEE Control Systems Letters, Transactions of the Institute of Measurement and Control, Control Theory and Technology, Mathematical Problems in Engineering, Unmanned Systems, and the International Journal of Automation and Computing. 